\title{
A Score Level Fusion Method on Fingerprint and Finger Vein
}

\author{
Jian Peng ${ }^{1}$, Jingyi $\mathrm{Wu}^{2}$ and Yun $\mathrm{Chen}^{2}$ \\ ${ }^{1}$ Nanjing University of Posts and Telecommunications, College of Overseas Education, Nanjing, Jiangsu, China \\ ${ }^{2}$ Nanjing University of Posts and Telecommunications, Science College, Nanjing, Jiangsu, China
}

\begin{abstract}
In this paper, we represent a score level fusion method on fingerprint and finger vein. Each unimodal identification system carries out processes of image preprocessing, feature extraction and feature matching to generate a vector of score. And we apply clustering analysis to split the score range into zones of interest. Then a decision tree and weighted-sum approach are used to make the decision. We test the proposed method on standard biometric database. Three metrics, namely, False Accept Rate, False Reject Rate, Recognition Rate, are used to evaluate experimental results. And experimental results show that the fusion system has a better performance than unimodal identification system.
\end{abstract}

\section{Introduction}

Biometric identification system is a prerequisite to ensure the security of the system. But it is difficult to realize an extreme high recognition rate system with unimodal identification system. Unimodal system which is based on single modality has several inherent problems like intra-class variation, spoofing attacks and failure-to-enroll rate. In this situation, fusion process helps to solve these problems. A multibiometric system is the system which fuse different single biosignature together to do identification [1]. There are different kinds of fusion methods which are sensor level fusion, feature level fusion, score level fusion and decision level fusion [2].

Score level fusion has advantages that have a wide application for varieties of modalities and score level carries relatively rich information about original images [3]. There are two approaches for score level fusion. The combination means combining the scores from matching to form a single score to make the final decision [4].

In this paper, we work on the fusion on the score level for fingerprint and finger vein. Each modality has its own identification system and each of the system will generate a vector of scores [5]. The scores are composed of matching values.

Dividing score range into zones of interest and dealing with them will improve the performance of calculation [6]. Fusion of scores was achieved either by combination [7] or by classification [5]. In [8], it proposed the combination of these two methods which is called BCC. K-means algorithm is used to divide the score range into zones of interest [8]. However, the combined method did not give a convinced demonstration about how to allocate weight to each score from unimodal identification system. We use the method in [9] to improve this fusion approach. In this way, we improve the method further and it makes this algorithm more complete.

For each unimodal identification, we also make a detailed design and try to ensure high recognition rate. In terms of fingerprint, traditional algorithm is used in fingerprint identification subsystem. In [19], Poincare Index algorithm is used to extract core points according to Crossing Number $(\mathrm{CN})$ theory, extract minutia points (ridge end points and bifurcation points), reduce the endpoint through continuous smooth processing, and finally obtain enough feature points. In [10], fingerprint image matching results are calculated according to the distance matching algorithm. Then we will get the scores of matching values which range 0 to 1 . And the closer the score is to 0 , the more accurate the matching result will be. The above are the methods for fingerprint identification system. In terms of finger vein, several image processing and image matching methods were applied. The fuzzy logic theory [13] is developed in the step of the vein segmentation. the problem of vein features extraction with two-dimensional Gabor filter is solved in [14]. Finally, the Hamming distance HD [15] is calculated to obtained matching score which represents the matching degree of two images. Since the distance HD does not range 0 to 1 , it is necessary to do some normalization to make it range 0 to 1 [16].

After the generation of scores of matching values from each unimodal identification system, it will be the fusion process. In [8], the method achieves the classification by the decision tree combined to the weighted sum (BCC) in the paper. And it generates a new score level fusion method. However, in [8], it does not give a convincing demonstration about the allocation of weight and it just gives fixed weights to iris scores and fingerprint weight. As we all know, different data distributions should have different weights. Therefore, it is necessary to give a clear computing method on weights 
of scores. In [9], optimal weight of score level is illustrated in different angles. We do some research on it and choose the appropriate computing method which can be applied in the fusion process. In this way, we do not only use the weighted sum method to make the fusion method more effective, but also improve the fusion method which can be used among many other biometrics.

\section{Fingerprint identification}

The fingerprint identification is made of three parts which are image preprocessing, feature point extraction and feature point matching.

The first step is to scale the fingerprint image and transform the color image into gray image. The image format is modified for subsequent operation.

The second step is to normalize, so that its gray value is limited to a certain range.

The third step carries on the multi-region threshold segmentation [17], according to the sum of the eight neighborhood points to distinguish the foreground and background colors.

The fourth step is to remove the image noise by means of mean filtering [17], then adopt the enhancement method based on ridge direction field [17]. And binary image, finally enhance the fingerprint pattern along the ridge.

The fifth step is to remove the cavities and glitches in the fingerprint [10].

The sixth step is to refence the concepts of morphological operation [18]. The open operation and closed operation of the processed image are carried out to refine the images.

The main purpose of extracting fingerprint feature points is to calculate the feature information of core and minutia. Poincare Index algorithm [19] is used to extract the fingerprint core points. The algorithm is robust when dealing with image noise. When the Poincare Index value of a pixel is $\pi$, the pixel is determined to be the core point, and the coordinate and direction field information is extracted.

Feature point matching mainly adopts the method of matching about distance [17].

In this paper, we will find a feature point. Then, starting from this feature point, we will do a detection in a distance along the ridge. Every step of detection will be saved. After multiple detections, we will get the arrays with information about feature points and use Euclidean distance to do the comparison.

The comparison of fingerprints was performed by the Euclidean distance, given by Eq. (1).

$$
D=\sqrt{\sum_{k-1}^{n}\left(x_{k}-y_{k}\right)^{2}}
$$

\section{Finger vein identification}

The vein recognition system is made up of four main steps, including pre-processing, segmentation, feature extraction and vein matching.
The image pre-processing is used to normalize the geometry size and gray scale of the image referred to the concept of grey-scale interpolation [11]. There are lots of redundant information contained in the vein image which are detected by the bilinear interpolation method [12], then the Gabor filter is applied to separate the useful information from the redundant information and achieve the purpose of enhancing the image.

The vein segmentation requires threshold segmentation algorithm [12] which is based on fuzzy logic [13] to accomplish the binarization of the image. This step is used to divide the image into three regions, namely, the background area, the blur area and the foreground area, in order to remain as many significant vein features as possible, meanwhile, isolate the binary image.

The feature extraction is aimed to extract the phase and direction of the vein features based on the characteristic that the directional lines of vein, which was developed by applying two-dimensional Gabor filter [14], leading to a specific texture image. Then the direction characteristic code and phase characteristic code of the vein have been obtained.

Fusing the characteristic of phase and direction, the score for vein matching is gained based on calculating the Hamming distance HD [6] between the prototype vein code and the under-tested vein code, as the Eq. (2) shows:

$$
H D=\frac{\|(\operatorname{code} M \otimes \operatorname{code} N) \cap \operatorname{mask} M \cap \operatorname{mask} N\|}{\|\operatorname{mask} M \cap \operatorname{mask} N\|}
$$

The code $\mathrm{M}$ and code $\mathrm{N}$ are inferred from two finger vein images, while the mask $M$ and mask $N$ are the respective masks of the two images due to the blocked light.

\section{Score level fusion approach}

The output of fingerprint identification system and finger vein identification will be used for score level fusion. The score normalization can transform scores of different systems into a common domain before combing them $[15,27]$. We use Min-Max method to normalize the output of both identification systems. The equation is shown in Eq. (3).

$$
s_{i}^{*}=\frac{s_{i}-\min (s)}{\max (s)-\min (s)}
$$

In this paper, we use $\mathrm{BCC}$ in [8] to do the score level fusion and do some modification in the process of calculating weighted sum.

\subsection{Dividing the score range into zones of interest}

Our research is the fusion based on score level. Therefore, the score range is what we will deal with. We will divide the score range into zones of interest according to the numerical features of scores. After the dividing, numerical features of each zone will be more prominent. Then we can give these digital characteristics practical 
meaning for further processing which helps to improve efficiency of fusion. Hence, clustering analysis is an effective tool. It can classify scores of matching values according to numerical characteristics.

Based on the scores generated by each unimodal identification system, the score range will be divided into three zones zone 1, zone 2 and zone 3 [8]. Zone 1 is the certainty zone where the user is identified (identical class) if his identification score is in this zone. Zone 2 is the uncertainty zone where the identification is not sufficiently reliable (undefined class). Zone 3 is the certainty zone where the user is not identified (different class).

$\mathrm{K}$-means algorithm is used to divide the score range into zones into interest [8]. The advantages of K-means algorithm are the fast convergence and good clustering effect. And the effect of classification will be accurate with the dense sample data. First, we build the standard biometric database of each unimodal system. It will output group of scores of matching values through the processing of image preprocessing, feature extraction and feature matching. According to different decision thresholds from 0 to 1 , we calculate the FAR (False Accept Rate), and FRR (False Reject Rate). Using FAR and FRR as the coordinate in which FAR is abscissa and FRR is ordinate, we plot the ROC curve and calculate the EER. EER is for further discussion. The set of coordinates from ROC curve is the input of $\mathrm{K}$-means algorithm. The score range is divided into $\mathrm{K}$ zones $\mathrm{Z}=\left\{\mathrm{Z}_{1}, \mathrm{Z}_{2}, \ldots \mathrm{z}_{\mathrm{k}}\right\}$. Assuming that the center of a clustering is $\mathrm{m}_{\mathrm{i}}(\mathrm{FAR}, \mathrm{FRR})$, the point $\mathrm{x}_{\mathrm{j}}$ (FAR,FRR) will belong to zone $i$ if its distance to the center of the zone $\mathrm{i}$ is smaller than its distance to the center of any other zone.

To minimize the distance between $\mathrm{x}_{1}, \mathrm{x}_{2}, \ldots \mathrm{x}_{\mathrm{n}}$ in each zone, we choose the square error function as objective function in Eq. (4):

$$
V=\sum_{i=1}^{k} \sum_{x_{j} \in z_{i}}^{k}\left\|x_{j}-m_{i}\right\|^{2}
$$

After clustering the ROC curve, we get three zones. Finding the boundary value of decision threshold, and we can divide the score range into zones of interest. Threshold of dividing zone 1 and zone 2 for fingerprint is recorded as $\mathrm{P}_{1}$. And threshold of dividing zone 2 and zone 3 for fingerprint is recorded as $P_{2}$. Threshold of dividing zone 1 and zone 2 for finger vein is recorded as $\mathrm{V}_{1}$. And threshold of dividing zone 2 and zone 3 for finger vein is recorded as $V_{2}$.

\subsection{Score level fusion method based on classification and weighted sum}

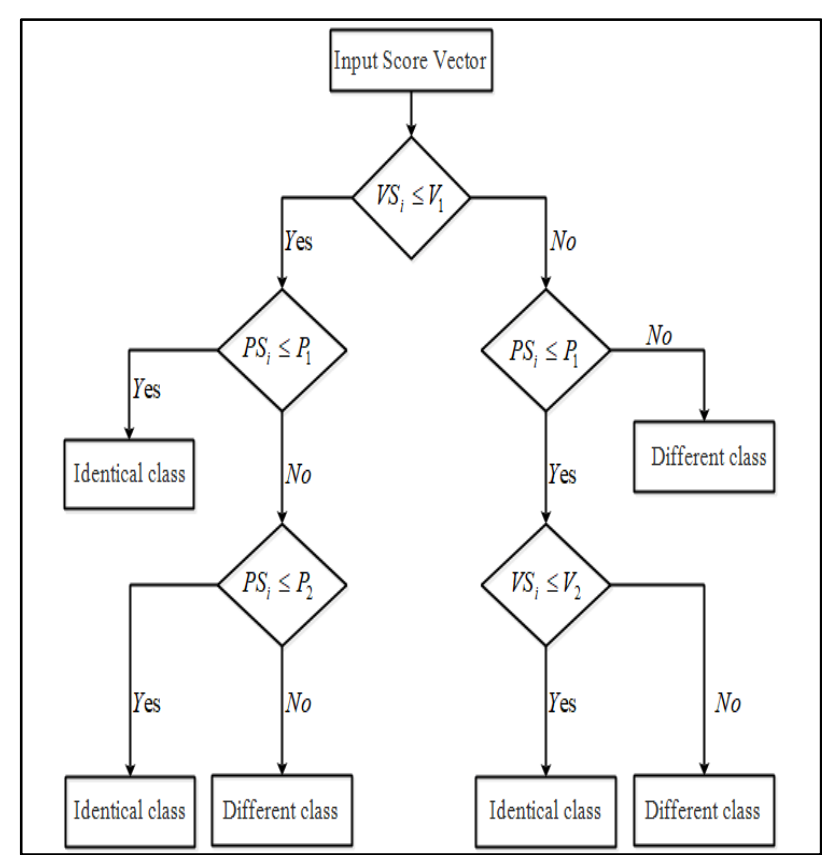

Fg.1 Decision tree of classification

The input is a group of 2-D vectors of scores which is composed of the users' fingerprint and finger vein score of matching values obtained by fingerprint and finger vein identification system. The decision tree based on the zones of interest shown in Fg.1 in [8] will classify the scores. The flowchart shown in Fg.2 in [8] will do the final decision according to the results of classification.

Decision tree makes decision according to scores of matching values and thresholds. The purpose is to distinguish the vector of scores that can be recognized and the one that cannot be recognized. In the flowchart, 


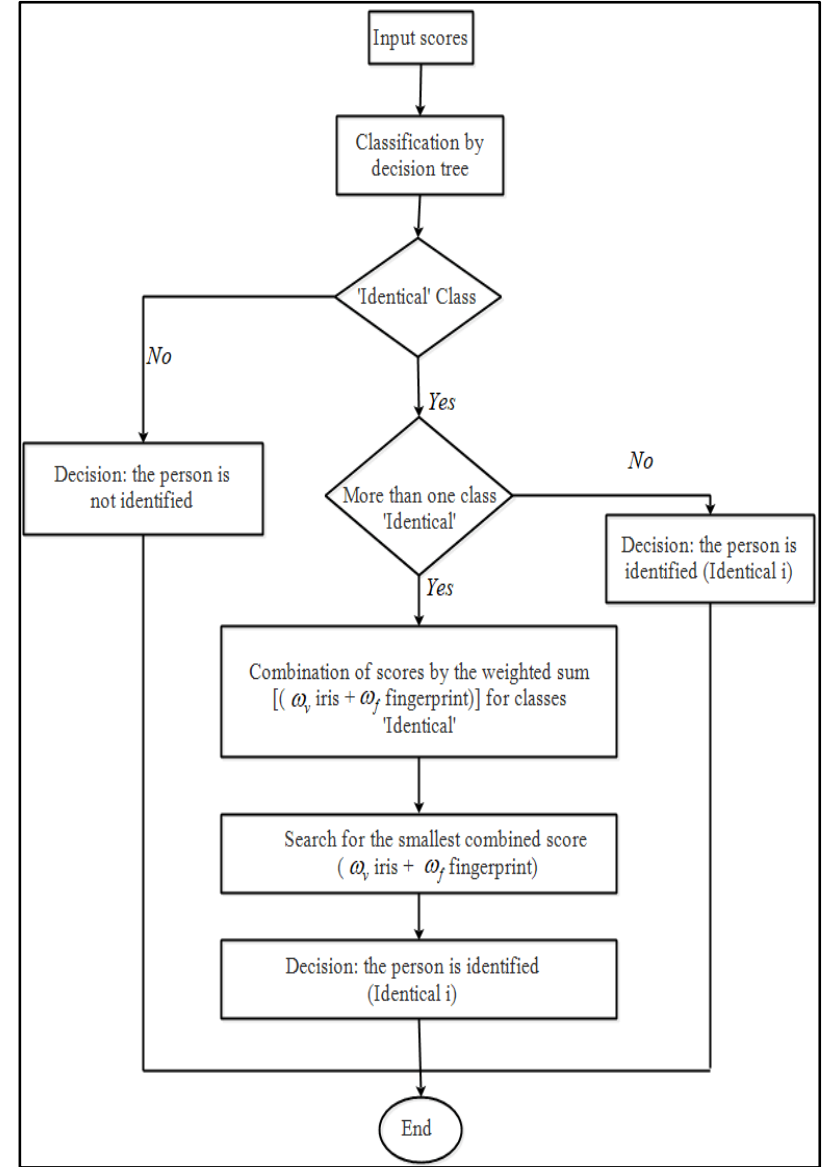

Fg.2 Flow chart of combination

if the result of classification is Different Class for all of vectors of scores, the person is not identified. If only one vector of score is identical class, the identity of the user will be the identity of the corresponding user of this vector of score. If several vectors of scores are identical class, weighted sum of each vector of score will be calculated. Find the minimal sum, and the identity of the user will be the identity of the user which the minimal sum corresponding to.

However, in the stage of weighted sum, paper [9] does not give a convincing demonstration about how to allocate weight to each score from unimodal identification system. We use the method in [9] to improve this fusion approach. EER will be minimal in this condition which is shown in Eq. (5) and Eq. (6):

$$
\begin{aligned}
& W_{1}=\frac{\sigma_{i m p, 2}^{2}+\sigma_{g e n, 2}^{2}}{\sigma_{i m p, 1}^{2}+\sigma_{g e n, 1}^{2}+\sigma_{i m p, 2}^{2}+\sigma_{g e n, 2}^{2}} \\
& W_{2}=\frac{\sigma_{i m p, 1}^{2}+\sigma_{g e n, 1}^{2}}{\sigma_{i m p, 1}^{2}+\sigma_{g e n, 1}^{2}+\sigma_{i m p, 2}^{2}+\sigma_{g e n, 2}^{2}}
\end{aligned}
$$

In these two equations, $\sigma_{i m p, 1}^{2}$ and $\sigma_{g e n, 1}^{2}$ are respectively variances of $X_{i m p, 1}$ and $X_{g e n, 1} \cdot \sigma_{i m p, 2}^{2}$ and $\sigma_{g e n, 2}^{2}$ are respectively variances of $X_{i m p, 2}$ and $X_{g e n, 2} \cdot X_{i m p, 1}$ is the set of scores of matching values in intra-class test for modality 1 . $X_{\text {gen }, 1}$ is the set of scores of matching values in inter-class test for modality 2 . It is the same with modality 2 . It is clear that $X_{1}=X_{i m p, 1}+$ $X_{g e n, 1}$ and $X_{2}=X_{i m p, 2}+X_{g e n, 2}$.

\section{Experiment and results}

In the experiment, we use two standard biometric databases: FVC2002_DB1_B for fingerprint and FV-USM for finger vein. On the base of the two standard biometric databases, we build double modality database which contains 50 users. The identities of users are decided by the combination of their fingerprint and finger vein signatures. There are 5 images for each modality from each user. For each modality from each user, 3 images are stored in database as samples. The other 2 samples are one for training and one for testing. It means that 50 images are used to calculate zones of interest and 50 images for evaluation.

To evaluate the performance of this fusion system, we calculate FAR, FRR. Meanwhile, Recognition Rate is also calculated according to FAR, FRR and thresholds for the evaluation. We use K-means algorithm to divide the score rang into zones of interest and plot ROC curve through K-means clustering analysis. The curves are shown in Fg.3 and Fg.4.

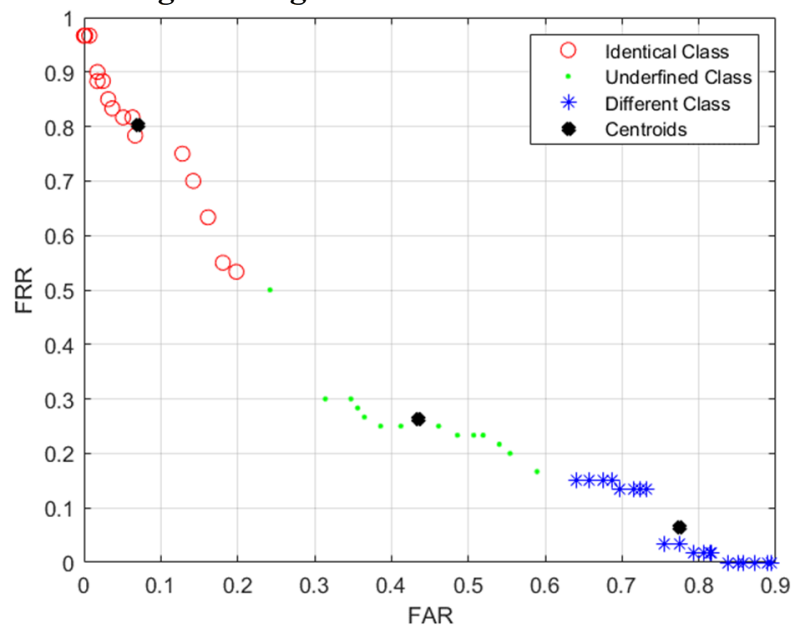

Fg.3 ROC curve of fingerprint through K-means clustering analysis

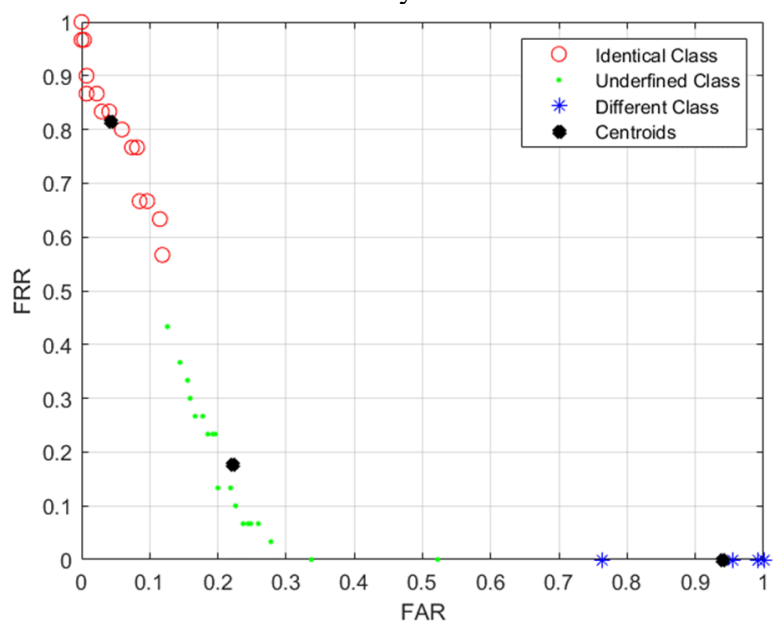

Fg.4 ROC curve of finger vein through K-means clustering analysis 
Meanwhile, the calculation in background program of thresholds which are $\mathrm{P}_{1}, \mathrm{P}_{2}, \mathrm{~V}_{1}$ and $\mathrm{V}_{2}$ of zones of interest is completed. Thresholds are shown in Table1.

Table 1 Thresholds for fingerprint and finger vein

\begin{tabular}{l|ll}
\hline Threshold & $P_{1} / V_{1}$ & $P_{2} / V_{2}$ \\
\hline Fingerprint & 0.33 & 0.49 \\
Finger vein & 0.59 & 0.89 \\
\hline
\end{tabular}

After calculating these thresholds, we will use the testing sets to apply the modified fusion method to score level fusion between fingerprint and finger vein. The results of experiments are shown in Table 2.

Table 2 The performance of different systems

\begin{tabular}{l|lll}
\hline \multirow{2}{*}{ System } & \multicolumn{3}{|l}{ Rate } \\
\cline { 2 - 4 } & \multicolumn{3}{|l}{ FARFRRRR } \\
\hline Fingerprint System & $38.60 \%$ & $25.00 \%$ & $88.33 \%$ \\
Finger vein System & $21.85 \%$ & $13.00 \%$ & $90.67 \%$ \\
BCC & $15.37 \%$ & $12.79 \%$ & $91.56 \%$ \\
Modified BCC & $14.11 \%$ & $10.22 \%$ & $92.44 \%$ \\
\hline
\end{tabular}

In Table 2, we can find that, not only iris and fingerprint, but also finger vein and fingerprint are applicate for the score level fusion method BCC. Furthermore, our efforts in the modification on the method are worthwhile because compared to BCC, modified $\mathrm{BCC}$ reduces false accept rate and false reject rate and increase recognition rate.

\section{Conclusion}

In this paper, we work on the multibiometric fusion in score level for the identifications of fingerprint and finger vein. We choose the method which has been used in different types of biological modalities.

We use Hamming distance and Euclidean distance to calculate scores of matching values. Then, scores should be through the normalization. K-means algorithm is for the dividing of zones of interest. The decision tree for classification and flowchart for combination will achieve the score level fusion. Meanwhile, we choose one method for the calculation of weighted sum which is our modification on the method BCC.

In this way, we have one more train of thought to work on the fusion in the modality of fingerprint and finger vein. And our modification on the method BCC is effective. Furthermore, the modification not only improves the performance of the multibiometric fusion for fingerprint and finger vein, but also the calculation method of weight brings the method BCC to a whole. The results show that the method BCC is effective in the fusion of fingerprint and finger vein and our medication on BCC improves the performance of BCC.

Critically, K-means algorithm may not be suitable for varieties of distributions of scores of matching values. The choice of clustering analysis algorithm should be based on the actual situation about scores.

\section{Acknowledgements}

This work was supported in part by STITP, Nanjing University of Posts and Telecommunications, under Grant SYB2019032.

\section{References}

1. M. He, S.J. Horng, P. Fan, R.S. Run, R.J. Chen, J.L. Lai, M.K. Khan and K.O. Sentosa, Performance evaluation of score level fusion in multimodal biometrics systems, Pattern Recognition, Vol.43(5), 1789-1800 (2010)

2. M. Hanmandle, J. Grover, A. Gureja and H.M. Gupta, Score level fusion of multimodal biometrics using triangular norms, Pattern Recognition Letter, Vol.32(14), 1843-1850 (2011)

3. M. Heikkila, M. Pietikainen and C. Schmid, Description of interest regions with local binary patterns, Pattern Recognition, Vol.42(3), 425-436 (2009)

4. A. Jain, K. Nandakumar and A. Ross, Score normalization in multimodal biometric systems, Pattern Recognition, Vol.34(12), 2270-2285 (2005)

5. K. Aizi and M. Ouslim, A new multibiometric identification method based on a decision tree and a parallel processing strategy. Int.Rev. Comput.Softw.8(10), 2507-2514 (2013)

6. F. Wang and J. Han, Multimodal biometric authentication based on score level fusion using support vector machine, Opto-Electron. Rev.17(1), 59-64 (2009)

7. J.A. Shoa'a and A. Mananl, Biometric in health care security system, Iris-Face fusion system. Int.J.Acad. Res.3(1). 1-11 (2011)

8. K. Aizi and M. Ouslim, Score level fusion in multi-biometric identification based on zones interest, Journal of King Saud University Computer and Information Sciences, https://doi.org//10.1016/j.jksuci.2019.9.003

9. B. Zhou, X. Lin, H. Jia and Y. Zhou, Optimal weight of the score level multiple biometric feature fusion, $\mathrm{J}$ Tsinghua Univ (Sci \& Tech), Vol.48, No.2(2008)

10. L. Yang and Z. Li, Embedded Fingerpoint Indexing System Based on SEP6200, Microcontrollers\&EmbeddedSystems 2014, 6:47 (in Chinese)

11. MrCharles, Fingerprint Identification Source Code https://github.com/charlesLucky/FingerRecognitionF romScratch (2019)

12. Q.P. Huang and X.B. Wang, Computer Simulation, 05, 111-114 (2005)

13. M. Jerome and B. Azeddine, IEEE Transactions on Pattern Analysis and Machine Intelligence, 21, 940-946, (1999)

14. J. Daugman, IEEE Transactions on Acoustic, Speech and Signal Processing, 7, 1169-1179, (1988)

15. K.J. Wang and Z. Yuan, Pattern Recognition and 
Artificial Intelligence, 10, 692-697, (2007)

16. W. Kabir, M.O. Ahmad and M.N.S. Swamy, Normalization and Weighting Techniques Based on Genuine-Impostor Score Fusion in Multi-Biometric Systems, IEEE Transactions on Information
Forensics and Security, Vol.13(8), 1989-2000 (2018)

17. A. Noor, ANew Algorithm for Minutiae Extraction and Matching in Fingerprin, Doctor thesis, London: Brunel University, (2012) 